\title{
Is the Fast Evolution Scenario for Virialized Compact Groups Really Compelling? The Role of a Dark Massive Group Halo
}

\author{
M.A. Gómez-Flechoso ${ }^{1}$ and R. Domínguez-Tenreiro \\ Dpt. Física Teórica C-XI, Universidad Autónoma de Madrid, \\ E-28049 Cantoblanco, Madrid, Spain
}

Received _ ; accepted

\footnotetext{
${ }^{1}$ Present address: Observatoire de Genève, Ch. des Maillettes 51, Ch-1290 Sauverny (Switzerland)
} 


\begin{abstract}
We report on results of N-body simulations aimed at testing the hypothesis that galaxies in X-ray emitting (i.e., virialized) Compact Groups are not tidally stripped when they are embedded in a common, massive, quiescent dark matter halo. To disentangle the effects of interactions from spurious effects due to an incorrect choice of the initial galaxy model configurations, these have been chosen to be tidally-limited King spheres, representing systems in quasi-equilibrium within the tidal field of the halo. The potential of the halo has been assumed to be frozen and the braking due to dynamical friction neglected. Our results confirm the hypothesis of low rates of tidal stripping and suggest a scenario for virialized Compact Group evolution in their quiescent phases with only very moderate tidally induced galaxy evolution can be generally expected. This implies the group stability, provided that the dynamical friction timescales in these systems are not much shorter than the Hubble time. We discuss briefly this possibility, in particular taking account of the similarity between the velocity dispersions of a typical virialized Compact Groups and the internal velocity dispersion of typical member galaxies. A number of puzzling observational data on Compact Groups can be easily explained in this framework. Other observations would be better understood as the result of enhanced merging activity in the proto-group environment, leading to virialized Compact Group formation through mergers of lower mass halos, as predicted by hierarchical scenarios of structure formation.
\end{abstract}

Subject headings: galaxies: structure — galaxies: interactions — galaxies: kinematics and dynamics 


\section{INTRODUCTION}

Compact groups (CG) of galaxies are relatively isolated systems composed of a small number of galaxies (three or more) forming configurations with very low median projected intergalactic separation, providing the highest projected galaxy number density systems in the sky. Hickson (1982) has pioneered the use of specific, quantitative selection criteria to create CG catalogs. Other catalogs now available include the Southern CG catalog (Prandoni, Iovino \& MacGillivray 1994), and those produced from the CfA2 (Barton et al. 1996) and Las Campanas (Allam \& Tucker 1998) redshift surveys.

Groups in Hickson's catalog and their member galaxies have been observed at many wavelengths and the extensive available data pose a number of challenging questions. Their high galaxy number density and relatively low velocity dispersion would imply, on theoretical grounds, short dynamical timescales and rapid evolution, making CGs the ideal sites for galaxy tidal interactions (and, eventually, tidal disruption) and mergers to occur. These would result in the appearance of more frequent dynamical peculiarities in CG galaxies, as well as enhanced star formation rates and nuclear activity compared with galaxies in less dense environments. Enhanced galaxy merging activity would produce merger remnants and would lead to the formation of blue luminous ellipticals in a time scale of $\sim 1$ Gyr. Tidal disruption would cause, eventually, the CG disappearance in a few crossing times ( $\sim 1.5$ Gyr; Barnes 1989). However, observations of Hickson Compact Groups (HCGs) do not support these predictions. A fraction as high as $43 \%$ of galaxies in HCGs may be interacting (Mendes de Oliveira \& Hickson 1994), but no correlation has been found between the number of interacting galaxies in a given group and the group global parameters (velocity dispersion, crossing times, X-ray properties; Mendes de Oliveira \& Hickson 1994; Pildis, Bregman \& Schombert 1995). Also, a high fraction of spirals in HCGs have asymmetric and peculiar rotation curves, but these dynamical 
peculiarities do not correlate with the CG global properties, even if the fraction of galaxies with peculiar rotation curves is higher among those belonging to CGs with lower galaxy velocity dispersion inside the group, $\sigma_{\text {group }} \leq 100 \mathrm{~km} \mathrm{~s}^{-1}$ (Nishiura et al. 2000). The $H \alpha$, $\mathrm{CO}$ and FIR observations of the HCG galaxies have shown neither enhancement in their present-day star formation rates nor in their star formation histories, when compared with control samples of field galaxies (see Verdes-Montenegro et al. 1998; Iglesias-Páramo \& Vílchez 1999 and references therein). Coziol et al. (1997), Coziol, Iovino \& de Carvalho (2000) and Allam et al. (1999) have found depressed star formation, relative to control samples, in CGs of the Hickson, Southern and Las Campanas catalogs, respectively, and, also, a density-morphology-activity relation in both HCGs and Southern CGs. Moreover, even if the fraction of galaxies in HCGs that are merger remnants is still controversial, observations suggest that it is not high (only 7\% in Zepf 1993; see also Hickson 1997 and references therein). In HCGs, the first ranked galaxy sample and the global population have the same fraction of E or S0 galaxies (Hickson 1982). Observations also suggest that there are not more blue ellipticals in HCGs than in other environments (Zepf \& Whitmore 1991). HCGs have a relatively large spiral fraction, but it is anticorrelated with the galaxy velocity dispersion (Hickson, Kindl \& Huchra 1988) and is lower in X-ray luminous CGs (these two parameters are, on their turn, correlated, with X-ray detected CGs having $\sigma_{\text {group }} \geq 100 \mathrm{~km} \mathrm{~s}^{-1}$, see Mulchaey 2000). These results and the HCG number density are in contradiction with the theoretical framework of rapid evolution, supported by the first N-body simulations on CG evolution (Barnes 1985, 1989; Mamon 1987). To surmount these difficulties, some authors have pointed out that a large fraction of HCGs are not real dense configurations, but chance superpositions of galaxies (Mamon 1986; Diaferio, Geller \& Ramella 1994; Hernquist, Katz \& Weinberg 1995, but also see Hickson \& Rood 1988). But this scenario does not account for the existence of a diffuse X-ray emission from hot intergroup gas in 75\% HCGs (Ponman et al. 1996). This gas has been found to 
have almost the same temperature in HCGs where it has been detected $\left(T_{\mathrm{X}} \simeq 0.9 \mathrm{keV}\right.$, with a detection limit of $T_{\mathrm{X}} \simeq 0.3 \mathrm{keV}$ ). The projected gas density profiles are consistent with $\Sigma_{\text {gas }}(s)=\Sigma_{\text {gas }}^{0} /\left[1+\left(s / R_{c}\right)^{2}\right]^{\left(3 \beta_{\text {gas }}-1\right) / 2}$, with core radius $4 \leq R_{c} \leq 30 h^{-1} \mathrm{kpc}$ and slope $0.38 \leq \beta_{\text {gas }} \leq 0.92$ for HCGs (see Mulchaey et al. 1996 and references therein). Assuming hydrostatic equilibrium ( $\beta$-model, see Cavaliere 1973), these observations imply a total group binding mass of $M_{\text {group }} \simeq 2 \times 10^{13} \mathrm{M}_{\odot}$ at $R \sim 300 \mathrm{kpc}$ for groups of the HCG catalog (see Mulchaey et al. 1996, and references therein), implying that galaxies in these CGs are embedded in a common massive, concentrated dark matter halo.

We present in this Letter a new framework to understand X-ray emitting CGs of galaxies as stable systems against tidal disruption of their galaxies, in the quiescent phases of their evolution . The main hypothesis is that the common virialized massive concentrated dark matter halo, in which galaxies are embedded in CGs, stabilizes both the group and the individual galaxies, so they are able to remain in dynamical equilibrium during many group crossing times after halo formation. This possibility has been tested through a series of N-body simulations, whose results confirm this assumption.

\section{A SCENARIO FOR STABLE CGS OF GALAXIES}

\subsection{Description of the simulations}

To study the stability of the galaxies in CGs and the group itself, we have built initial model galaxies and left them to orbit in the external halo potential. We have considered that the massive dark matter halos in which galaxies are embedded are consistent with the

\footnotetext{
${ }^{1}$ In any hierarchical scenario for structure formation, violent (i.e., merging) phases and quiescent phases follow each other in the evolution of any astronomical system. CGs halos would have been formed through merging of lower mass halos.
} 
gas distribution ( $\beta$-model) as explained above. A statistical analysis of the distribution of projected galaxy pair separations in accordant redshift quartets of HCGs by Montoya et al. (1996) shows that galaxy density profiles are consistent with those of the X-ray emitting gas (see above), with slope parameter $\beta_{\text {gal }}$ instead of $\beta_{\text {gas }}$. In this way, the galaxy and halo mass distributions are determined by four parameters among the following: $M_{\text {group }}, R_{c}$, $\beta_{\text {gas }}, T_{\mathrm{X}}$ (given by X-ray data), slope of the galaxy distribution profile, $\beta_{\text {gal }}$, and galaxy velocity dispersion inside the group, $\sigma_{\text {group }}$. We recall that, solving the Jeans equation, the $\beta$-model predicts that $\beta_{\text {spec }}=\sigma_{\text {group }}^{2} /\left(k T_{\mathrm{X}} / \mu m_{p}\right)=\beta_{\text {gas }} / \beta_{\text {gal }}$, and indeed this is the case for HCGs (Montoya et al. 1996). To illustrate the stabilizing role of massive halos, results for two different halo masses are reported: CG1 group model, with $M_{\text {group }}=1.85 \times 10^{13} \mathrm{M}_{\odot}$, and CG2 group model, with $M_{\text {group }}=2.78 \times 10^{12} \mathrm{M}_{\odot}$ at $R=300 \mathrm{kpc}$. The core radius of the group is $R_{c}=24 \mathrm{kpc}$ (Mulchaey et al. 1996). The remaining parameters of the halos (Table 1) are also consistent with those of HCGs, taking $H_{0}=50 \mathrm{~km} \mathrm{~s}^{-1} \mathrm{Mpc}^{-1}$ (Hickson et al. 1992).

The initial positions and velocities of the galaxies in the halo, $R_{i, \text { in }}$ and $V_{i, i n}$, $i=1,2, \ldots, \mathrm{N}_{\mathrm{G}}\left(\mathrm{N}_{\mathrm{G}}\right.$ is the number of galaxies in the group; as very often accordant HCGs are quartets, we have taken $\mathrm{N}_{G}=4$ ) have been assigned in the following way: (a) the positions are Monte-Carlo realizations of the average HCG galaxy number density profile found by Montoya et al. (1996), and (b) the velocities are assigned randomly with the condition that $\sum_{i=1}^{\mathrm{N}_{\mathrm{G}}} V_{i}^{2}=\mathrm{N}_{\mathrm{G}} \sigma_{\text {group }}^{2}$. The $R_{i, i n}, V_{i, i n}$ and the orbital angular momentum, $L_{i}$, are given in Table 2. Two sets of galaxy initial positions and velocities are reported for the massive CG1 group model; for one of them (CG1M), they have been prepared in such a way that they lead to a binary merger event of two galaxies.

Galaxies have been assumed to be self-gravitating configurations in quasi-equilibrium 
with the environment, that is, with the tidal field of the halo 2. As the simplest choice for the initial galaxy model, we assume that they are spheres with an isotropic velocity dispersion tensor. In this case, the tidal field determines the limiting or tidal radius, $r_{\mathrm{t}}$, of the configuration (King 1962; Gómez-Flechoso \& Domínguez-Tenreiro 2001, hereafter GD01), leading to the so-called tidally-limited (t-limited) King spheres, based on the King-Michie velocity distribution function (King 1966; Michie 1963). These $t$-limited King spheres are characterized by two free parameters among the total galaxy mass, $M_{\text {gal }}$, the 1 -dimensional internal velocity dispersion, $\sigma_{\text {gal }}$, the galaxy core radius, $r_{0}$, and the central potential, $W_{0}$ (see GD01 for details). Note that the widely used standard King spheres, whose limiting radius is left free (and not, as due, fixed by the tidal field), have instead three free parameters. In GD01 we describe a method to build $t$-limited King spheres with prefixed values of $M_{\text {gal }}$ and $r_{0}$. The initial configurations for our CG galaxies are Monte-Carlo realizations of the $t$-limited King spheres, with $\mathrm{N}_{\mathrm{p}}=10000$ particles, $M_{\text {gal }}=1.1 \times 10^{11} \mathrm{M}_{\odot}$ and $r_{0}=0.2 \mathrm{kpc}$. In Table 2 we give the tidal radius, $r_{t}$, and the $\sigma_{\text {gal }}$ and $W_{0}$ parameters corresponding to each galaxy model of the CGs reported in this Letter. The values of these parameters are within their observational range. These CG models have been left to evolve during a time interval of $10^{10}$ years $\left(\simeq 10^{2}\right.$ group crossing times). Evolution has been followed by integrating the equation of motion for

\footnotetext{
${ }^{2}$ Note that to properly quantify environmental effects, it is necessary that the initial model represents a galaxy in quasi-equilibrium within the tidal field. Otherwise, it would be difficult to disentangle between the effects that are effectively due to interactions and those that are spurious, due to an incorrect choice of the initial galaxy configuration, see Gómez-Flechoso and Domínguez-Tenreiro (2001b, in preparation) for a discussion. In fact, galaxy infall in numerical simulations of small galaxy group dynamics is mostly caused by (spurious) tidal heating, enhanced in some cases by two-body numerical heating.
} 
each constituent particle in the combined potential of the other particles and the halo by means of a vectorized version of the treecode (Hernquist 1987). As we are interested in galaxy stability against tidal disruption, the halo potential has been taken to be smooth, so dynamical friction effects have not been considered. However, no important dynamical friction effects are expected (see $\S 3$ ).

\subsection{Results}

For the massive type CG1 group, both the individual galaxies and the group itself are stable against tidal stripping, therefore very low tidally induced evolution can be expected in any of them. By galaxy stability we mean that: (a) the individual galaxies do not lose appreciably mass, as the fraction of mass stripped by tidal forces is low $(5-25 \%$, depending on the galaxy initial position), (b) the mass density profiles of the individual galaxies do not change, and, (c) the velocity distribution function of each galaxy does not evolve significantly, and, therefore, their internal velocity dispersion, $\sigma_{\text {gal,i, }}$, is almost constant; only a mild velocity anisotropy is developed in each galaxy. The CG does not change either, that is: (a) galaxies do not end up at the center of the configuration; on the contrary, the values of the galaxy pericentric and apocentric distances, as well as the median intergalactic separation, remain stable (see Figure 1a), and, (b) the galaxy velocity dispersion of the group, $\sigma_{\text {group }}$, does not change on average (Figure 1b). The same qualitative results have been found for the CG1M group, where the merger of the A and C galaxies soon after the begining of the simulation does not affect either the dynamical properties of the other galaxies in the group, nor the overall group evolution (see Figure 1).

All these results are mostly determined by the choice of the initial galaxy model configurations with an average density consistent with the halo density at galaxy pericenter, that is, they are tidal quasi-equilibrium solutions in the tidal field, and they are roughly 
independent on the galaxy or halo density profiles (see GD01 for details).

As $M_{\text {group }}$ decreases, binary interactions become more important relative to the overall halo potential. As a consequence, galaxies orbiting inside this halo become more likely to be tidally stripped and lose mass, and they tend to fall towards the halo center, where their remnants eventually end up, merging with each other and causing the group tidal disruption. An example of such a process is provided by the evolution of the low halo mass CG2 group model, where half of the mass inside $100 \mathrm{kpc}$ is in the galaxies. The galaxies of the CG2 group lose $30-50 \%$ of their mass during the first 4 Gyrs of the simulation. Their remnants merge and fall to the halo center. Figure 1 illustrates the group disappearance as a consequence of these mass loss. Taking more massive galaxies in this last experiment would result in a faster group disappearance, because the determining point here is the importance of the galaxy-galaxy interactions relative to the galaxy-halo interactions.

\section{SUMMARY AND DISCUSSION}

According to current scenarios of hierarchical structure formation, dark matter halos form through violent merger events from previously formed lower mass halos. These halos host baryonic clumps (either normal galaxies, dwarfs, and possibly HI clouds) that survive the halo merging and virialization, and, after this process is completed, they orbit inside the newly formed halo (Navarro, Frenk \& White 1995, hereafter NFW; Tissera \& Domínguez-Tenreiro 1998). In the present Letter, we show that very little mass is stripped from typical galaxies orbiting inside the potential well of a quiescent, massive CG-like halo, provided that the halo mass is similar to that inferred from observations of CGs with diffuse X-ray emission and that the initial galaxy models are self-gravitating configurations in

quasi-equilibrium within the tidal field of the halo. When these two conditions are met, the rate of tidal stripping is roughly independent of the galaxy or halo density profile, as shown 
by GD01, where a cuspy halo density profile as those obtained by NFW has been adopted. So, profiles do not need to have low concentrations, as demanded by Athanassoula, Makino \& Bosma (1997). These authors used standard (as opposed to $t$-limited) King spheres as initial model galaxies, that suffer from important mass loss unless that the CG halos they orbit are almost homogeneous. An important consequence of the tidal quasi-equilibrium in astronomical systems is that dynamical timescales for galaxy infall in CGs, and the ensuing CG disappearance, are not set by mass loss due to tidal stripping.

Among other processes causing galaxy infall to the center of the configuration the most efficient is dynamical friction, whose timescales, $t_{\text {fric }}$, however, are difficult to predict. The well known Chandrasekhar formula gives, for a $\sim 2 \times 10^{13} \mathrm{M}_{\odot} \mathrm{NFW}$ halo and $\mathrm{a} \sim 10^{11}$ $\mathrm{M}_{\odot}$ galaxy initially placed at $\sim 40$ - $90 \mathrm{kpc}$ from the halo center, a value of $t_{\text {fric }} \sim 3$ 6 Gyrs (Klypin et al. 1999). This situation corresponds roughly to the virialized CGs described in this paper. Domínguez-Tenreiro \& Gómez-Flechoso (1998, hereafter DG98) have shown that if the internal velocity dispersion of the orbiting galaxies is of the order of $\sigma_{\text {group }}$, then $t_{\text {fric }}$ could easily be a factor of $\sim 2$ - 3 longer than the values above, and even much more longer, depending very strongly on the particular values of the GC halo and galaxy parameters. Unfortunately, these analytical estimations of $t_{\text {fric }}$ are difficult to test through numerical simulations of the evolution of CGs with live halos. The main pitfall is the correct implementation in the simulations of the ratio between the fluctuating forces (causing dynamical friction) and the smooth forces, $R_{\mathrm{fs}}$, that must be low to accurately simulate the dynamical friction effects. And so, the proper sampling of a virialized CG-like halo would demand a too high number of particles for current computer facilities. If $R_{\mathrm{fs}}$ is unphysically high, it results into undesired numerical effects, leading to an overestimation of the fluctuating force intensity and, consequently, to a too low $t_{\text {fric }}$ value. This is the so-called discreetness effect (see Hernquist \& Weinberg 1989; see also Eq. (14) in DG98). The simulations of CG evolution by Bode, Cohn \& Lugger (1993) suggest that $t_{\text {fric }}$ is roughly 
consistent with Chandrasekhar's formula predictions; however, these simulations could suffer from a discreetness problem, as they use a too low number of particles per galaxy and halo. In conclusion, we see that even if the case for inefficient dynamical friction in CGs is not definitively closed, analytical estimations of $t_{\text {fric }}$ suggest that, on theoretical grounds, the need of a rapid evolution scenario for virialized CGs becomes rather questionable.

A scenario where virialized CGs are dynamically quiescent since the end of the violent relaxation phase ensuing the merger episode that formed their massive dark matter halos could explain a number of difficulties met by the rapid evolution scenario (see $\$[1$ ).

First, the lack of clear correlations between the rate of galaxy interactions and CG global parameters is naturally explained if the galaxies are embedded in a common massive halo that determines their trajectories. The galaxy-galaxy binary interactions only represent perturbations to the overall halo potential and they are uncorrelated with global CG parameters. As shown for the CG1M group, if one such binary interaction occurs, the remaining CG galaxies are completely unaffected by this process. Binary interactions do destroy CGs if they are important relative to the global field of forces caused by the halo, that is, if the common halo is not massive enough compared with the mass of individual galaxies (the CG2 group). So, a range of CG halo masses could be responsible for the different degrees of dynamical evolutionary stages of CGs found by Ribeiro et al. (1998). But these could also result from different degrees of virialization after the merging event involving galaxy halos and leading to the common massive dark CG halo.

Second, the lack of enhanced merger activity inside quiescent CG halos found in our simulations suggests that CG early-type galaxies are unlikely to form in the quiet phases. It explains the anticorrelation found between the spiral fraction in CGs and $\sigma_{\text {group }}$ or the X-ray luminosity. The CG early-type galaxies can be formed through the same merger trees responsible for CG halo formation in a proto-group environment. The hierarchical scenario 
predicts that the merger rates are higher in dense environments than in isolation (Evrard, Silk \& Szalay 1990; Tissera \& Domínguez-Tenreiro 1998; see also Vázquez-Semadeni 1994), where systems form earlier, and lead to more massive compact halos (CGs would be more likely to appear within looser groups than isolated, see Barton, de Carvalho \& Geller 1998) and to more early-type galaxies within more massive (or more X-ray luminous or with a higher $\sigma_{\text {group }}$, given the correlations among these quantities, see Mulchaey 2000) CG halos. These pre-virialization violent mergers could also have triggered important gas inflows in the baryonic systems, giving rise, at that time, to starburst galaxies, that evolved into low luminosity AGNs in the post-merger phase, as the gas supply was gradually exhausted. This could be the origin of the density-morphology-activity relation found by Coziol et al. $(1997,2000)$, difficult to explain in the framework of the replenishment scenario (Governato et al. 1996).

Finally, the lack of enhancement of star formation in the main body of CGs as compared to field galaxies can be seen as another consequence of the density-morphology-activity relation: starbursts would have been induced sometime in the past, and the faster evolving galaxy members, placed at the CG cores, are now observed in a quiet phase, as are early-type galaxies in galaxy clusters.

It is a pleasure to thank l'Observatoire de Genève for its kind hospitality while this work was finished. MAGF was supported by the Dirección General de Enseñanza Superior (DGES, Spain) through a fellowship. It also supported in part this work, grants AEN93-0673, PB93-0252 and PB96-0029. 


\section{REFERENCES}

Allam, S.S., \& Tucker, D.L. 2000, AN, 321, 101

Allam, S.S., Tucker, D.L., Lin, H., \& Hashimoto, Y. 1999, ApJ, 522, L89

Athanassoula, E., Makino, J. \& Bosma, A.1997, MNRAS, 286, 825

Barnes, J.E. 1985, MNRAS, 215, 517; 1989, Nature, 338, 123

Barton, E.J., Geller, M., Ramella, M., Marzke, R.O., \& da Costa, L.N. 1996, AJ, 112, 871

Barton, E.J., de Carvalho, R.R., \& Geller, M.J. 1998, AJ, 116, 157

Binney, J., \& Tremaine, S. 1987, Galactic Dynamics (Princeton: Princeton Univ. Press)

Bode, P.W., Cohn, H.N., \& Lugger, P.M. 1993, ApJ, 416, 17

Cavaliere, A. 1973, Mem. Soc. Ast. It., 44, 571

Coziol, R., Ribeiro, A.L.D., de Carvalho, R.R., \& Capelato, H.V. 1997, ApJ, 493, 563

Coziol, R., Iovino, A., \& de Carvalho, R.R. 2000, AJ, 120, 47

Diaferio, A., Geller, M.J., \& Ramella, M. 1994, AJ, 107, 868

Domínguez-Tenreiro, R., \& Gómez-Flechoso, M.A. 1998, MNRAS, 294,465 (DG98)

Evrard, A.E., Silk, J., \& Szalay, A.S. 1990, ApJ, 365, 13

Governato, F., Tozzi, P., \& Cavaliere, A. 1996, ApJ, 458, 18

Gómez-Flechoso, M.A., \& Domínguez-Tenreiro, R. 2001, ApJ, in press, astro-ph/0011432 (GD01)

Hernquist, L. 1987, ApJS, 64, 715 
Hernquist, L., Katz, N., \& Weinberg, D.H. 1995, ApJ, 442, 57

Hernquist, L., \& Weinberg, D.H. 1989, MNRAS, 238, 407

Hickson, P. 1982, ApJ, 255, 382; 1997, ARAA, 37, 357

Hickson, P., Kindl, E. and Huchra, J.P. 1988, ApJ, 331, 64

Hickson, P., \& Rood, H.J. 1988, ApJ, 331, L69

Hickson, P., Mendes de Oliveira, C., Huchra, J.P., \& Palumbo, G.G.C. 1992, ApJ, 399, 353

Iglesias-Páramo, J., \& Vílchez, J.M. 1999, ApJ, 518, 941

King, I.R. 1962, AJ, 67, 471;

King, I.R. 1966, AJ, 71, 64

Klypin, A., Gottlöber, S., Kravtsov, A.V., \& Khokhlov, A.M. 1999, ApJ, 516, 530

Mamon, G.A. 1986, ApJ, 307, 426

Mamon, G.A. 1987, ApJ, 321, 622

Mendes de Oliveira, C., \& Hickson, P. 1994, ApJ, 427, 684

Michie, R.W. 1963, MNRAS, 125, 127

Montoya, M.L., Domínguez-Tenreiro, R., González-Casado, G, Mamon, G.A. \& SalvadorSolé, E. 1996, ApJ, 473, L83

Mulchaey, J.S. 2000, ARAA in press, astro-ph/0009379 preprint

Mulchaey, J.S., Davis, D.S., Mushotzky, R.F., \& Burstein, D. 1996, ApJ, 456, 80

Navarro, J.F., Frenk, C., \& White, S.D.M. 1995, MNRAS, 275, 56 (NFW) 
Nishiura, S., Shimada, M., Ohyama, Y., Murayama, T., \& Taniguchi, Y. 2000, AJ, 120, 1691

Pildis, R.A., Bregman, J.N., \& Schombert, J.M. 1995, AJ, 110, 1498

Ponman, T.J., Bourner, P.D.J., Ebeling, H., \& Bohringer, H. 1996, MNRAS, 283, 690

Prandoni, I., Iovino, A., \& MacGillivray, H.T. 1994, AJ, 107, 1235

Ribeiro, A.L.B., de Carvalho, R.R., Capelato, H.V., \& Zepf, S.E. 1998, ApJ, 497, 72

Tissera, P.B., \& Domínguez-Tenreiro, R. 1998, MNRAS, 297, 177

Vázquez-Semadeni, E. 1994, ApJ, 423, 681

Verdes-Montenegro, L., Yun, M.S., Perea, J., del Olmo, A., \& Ho, P.T.P. 1998, ApJ, 497, 89

Zepf, S.E. 1993, ApJ, 407, 448

Zepf, S.E., \& Whitmore, B.C. 1991, ApJ, 383, 542 
Table 1. Group parameters

\begin{tabular}{ccccccc}
\hline \hline & $\beta_{\text {gas }}{ }^{\mathrm{a}}$ & $T_{X}$ & $R_{C}$ & $M_{\text {group }}(<300 \mathrm{kpc})$ & $\sigma_{\text {group }}$ & $\beta_{\text {gal }} \mathrm{b}^{2}$ \\
& & $(\mathrm{keV})$ & $(\mathrm{kpc})$ & $\left(\mathrm{M}_{\odot}\right)$ & $(\mathrm{km} / \mathrm{s})$ & \\
\hline $\mathrm{CG} 1$ & 0.56 & 1.0 & 24.0 & $1.85 \times 10^{13}$ & 252 & 1.4 \\
$\mathrm{CG} 2$ & 0.42 & 0.2 & 24.0 & $2.78 \times 10^{12}$ & 98 & 1.4 \\
\hline
\end{tabular}

${ }^{a}$ Typical values from X-ray observations (see Mulchaey et al 1996)

${ }^{\text {b}}$ Best-fit value by Montoya et al. (1996) 
Table 2. Galaxy parameters

\begin{tabular}{|c|c|c|c|c|c|c|c|c|c|c|c|c|}
\hline & \multicolumn{4}{|c|}{ CG1 } & \multicolumn{4}{|c|}{ CG1M } & \multicolumn{4}{|c|}{ CG2 } \\
\hline & $\mathrm{A}$ & B & $\mathrm{C}$ & $\mathrm{D}$ & $\mathrm{A}$ & B & $\mathrm{C}$ & $\mathrm{D}$ & $\mathrm{A}$ & $\mathrm{B}$ & $\mathrm{C}$ & $\mathrm{D}$ \\
\hline$\sigma_{\text {gal }}(\mathrm{km} / \mathrm{s})$ & 344 & 326 & 277 & 284 & 351 & 336 & 297 & 279 & 266 & 237 & 227 & 233 \\
\hline$r_{t}(\mathrm{kpc})$ & 8.4 & 9.6 & 14.5 & 14.2 & 7.6 & 8.9 & 12.0 & 14.6 & 16.6 & 20.7 & 22.4 & 22.1 \\
\hline$W_{o}$ & 7.3 & 7.5 & 8.2 & 8.1 & 7.2 & 7.4 & 7.9 & 8.1 & 8.3 & 8.7 & 8.9 & 8.8 \\
\hline$R_{i, i n}(\mathrm{kpc})$ & 32.7 & 42.6 & 72.6 & 88.1 & 6.8 & 38.8 & 48.6 & 85.7 & 29.7 & 38.7 & 45.8 & 52.5 \\
\hline$V_{i, i n}(\mathrm{~km} / \mathrm{s})$ & 497 & 465 & 234 & 495 & 327 & 489 & 186 & 618 & 181 & 79 & 253 & 107 \\
\hline$L_{i}(\mathrm{Mpc} \mathrm{km} / \mathrm{s})$ & 15.5 & 18.8 & 6.0 & 37.4 & 1.2 & 19.0 & 7.4 & 27.8 & 4.4 & 2.9 & 2.1 & 5.4 \\
\hline
\end{tabular}

Note. - The mass and the core radius of all the galaxies are $M_{\text {gal }}=1.1 \times 10^{11} \mathrm{M}_{\odot}$ and $r_{0}=0.2$ kpc, respectively. 


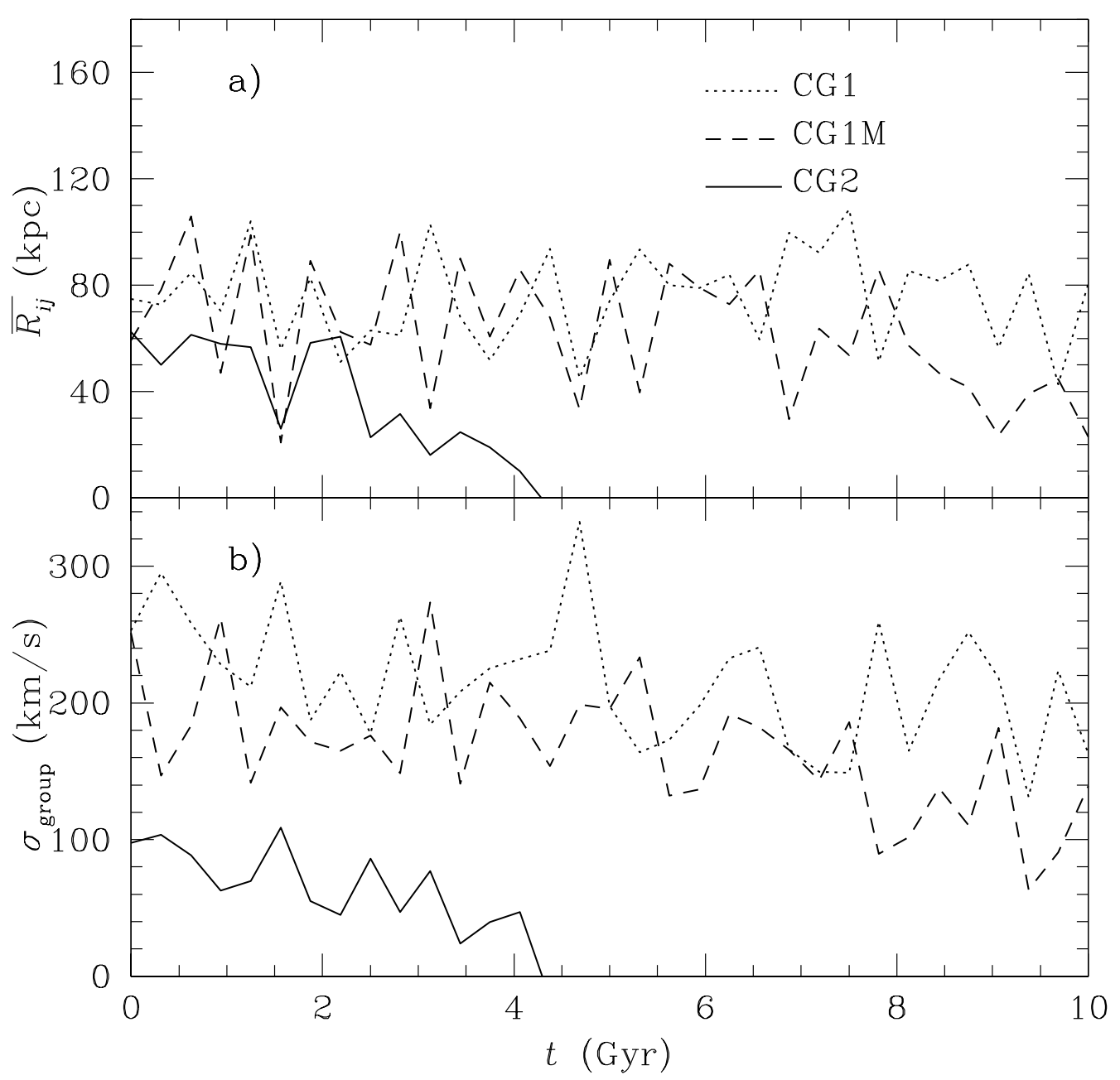

Fig. 1.- (a) Evolution of the median intergalactic separation and (b) the group velocity dispersion for the three group models. The merger of the remnants of A and C galaxy models in the CG1M group do not affect the evolution of these group global functions; by contrast, CG2 group disappearance is clearly seen in these plots. 